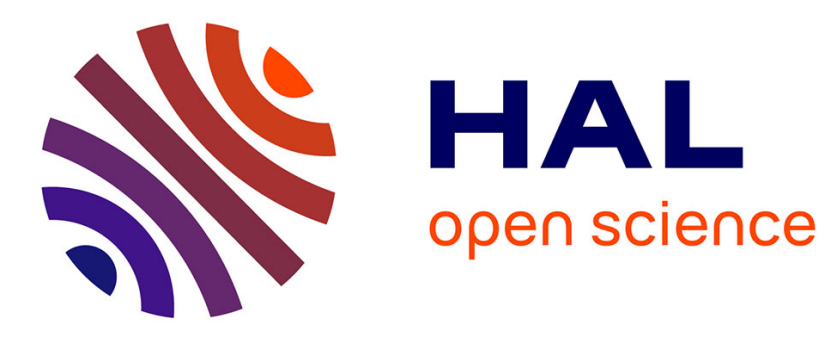

\title{
Collaboration in virtual worlds: the role of the facilitator
}

Benjamin Wigert, Gert-Jan de Vreede, Imed Boughzala, Ikram Bououd

\section{To cite this version:}

Benjamin Wigert, Gert-Jan de Vreede, Imed Boughzala, Ikram Bououd. Collaboration in virtual worlds: the role of the facilitator. HICSS '12: 45th Hawaii International Conference on System Science, Jan 2012, Maui, Hi, United States. pp.973 - 982, 10.1109/HICSS.2012.158 . hal-02408910

\section{HAL Id: hal-02408910 https://hal.science/hal-02408910}

Submitted on 13 Dec 2019

HAL is a multi-disciplinary open access archive for the deposit and dissemination of scientific research documents, whether they are published or not. The documents may come from teaching and research institutions in France or abroad, or from public or private research centers.
L'archive ouverte pluridisciplinaire HAL, est destinée au dépôt et à la diffusion de documents scientifiques de niveau recherche, publiés ou non, émanant des établissements d'enseignement et de recherche français ou étrangers, des laboratoires publics ou privés. 


\title{
Collaboration in Virtual Worlds: The Role of the Facilitator
}

\author{
Benjamin Wigert ${ }^{1}$, Gert-Jan de Vreede ${ }^{1,2}$, Imed Boughzala ${ }^{3}$, Ikram Bououd ${ }^{3}$ \\ ${ }^{1}$ The Center for Collaboration Science $\quad{ }^{2}$ Department of Systems Engineering $\quad{ }^{3}$ Telecom Business School \\ University of Nebraska at Omaha Delft University of Technology Institut TELECOM, France \\ \{bwigert,gdevreede\}@unomaha.edu, \{imed.boughzala,ikram.bououd\}@it-sudparis.eu
}

\begin{abstract}
Virtual worlds (VWs) are becoming a popular medium for meetings and collaborative problem solving efforts. However, complex VW communication tools and challenges in managing online social interactions are likely to complicate VW collaboration efforts. The purpose of our study therefore was to investigate the role of the facilitator when collaboration is conducted in a virtual environment. We developed a questionnaire based on major issues in real world collaboration and interviewed 14 subject-matter experts. Participants were asked to identify what key differences facilitators perceive between virtual and real world collaboration. In response, participants provided many insights, such as the new interpersonal management challenges that arise from the absence of face-to-face communication. Participants also warned of the challenges associated with the introduction of more technology to the collaboration process. Further, they identified credibility and trust issues that arise due to facilitators' avatar manipulation skills and avatar appearance. Suggestions for avoiding pitfalls and optimizing collaboration are provided.
\end{abstract}

\section{Introduction}

Over the past five years, VWs have evolved from being fancy chat rooms to virtual communities that support personal spaces, marketplaces, collaboration, and e-learning. With the emergence of such a dynamic work and play space, these activities can be conducted in real-time by people across the planet. In fact, a 2008 report conducted by Erica Driver and colleagues at Forrester Research, Inc. predicted that sometime between 2013 and 2015, VWs and the 3-Dimensional (3D) Internet will be as important to organizations as the Web is today. This research team also recommended that organizations begin establishing a presence in popular VWs. Undoubtedly, businesses can benefit from the marketing potential available in popular VWs, and even more so for the enhancement of essential business functions such as remote collaboration, personnel training, and the construction and sharing of 3D objects/artifacts [4]
Recently experts have echoed the assertions of Driver and colleagues (2008), suggesting that the collaborative capabilities inherent to VWs will change the landscape of how we interact on the Web by 2015 [6]. Notably, that does not mean that today's collaboration technology (e.g., video conferencing, text chat, Group Support Systems (GSS)) will be obsolete; rather, pertinent technologies will be integrated into VWs [5]. In many ways, the success of virtual world collaboration will be contingent on our ability to replicate real world activities in a VW such as remote collaboration. This activity is expected to yield some of the most significant business and societal implications $[1,4,5,9]$. VWs provide the traditional advantages of online communication in that they are convenient, efficient, allow synchronous communication for team members working at a distance, and reduce travel costs. Moreover, VWs add value to the virtual collaboration process in that they offer visual, aural, and spatial dimensions to the context of electronic communication [9, 14].

At present, Second Life is far and away the most frequented VW with its number of registered users jumping from over 2 million users in 2006 to over 21 million users in 2010 [2, 16]. Second Life provides an online 3D environment for friends, hobbyists, gamers, and employees to meet, interact and collaborate. Importantly, corporations are establishing their presence in Second Life both for purposes of marketing their products to Second Life users (i.e. residents) and to host internal and external collaborative projects. For example, major corporations such as Walt Disney, Nike, IBM, Cisco, Dell, National Aeronautics and Space Administration (NASA), and British Petroleum have capitalized on the reach of Second Life in regards to its massive international population and sophisticated collaboration tools [10]. Distributed teams can even take online corporate retreats in VWs. Alpine Executive Center (www.AlpineExecutiveCenter.com) provides corporate collaboration accommodations in the form of a venue located at a VW ski resort. Collaborators can enjoy interactive entertainment, such as skiing, sight-seeing, and art galleries, when they are not taking advantage of 
sophisticated decision making tools, such as brainstorming and voting tools, to efficiently and effectively host business meetings.

However, despite the many advantages to communicating in VWs, there is much to learn about how collaboration changes in these environments. Real-world collaboration is messy enough due to the cognitive and social challenges inherent to group work $[3,15]$. Thus, adding new technology, user competencies, and a change in the nature of user perceptions and communication makes collaboration that much more complex. In the real-world, many teams rely on facilitators to guide them through the difficulties of group work. However, the role and methods of facilitators is likely to change with the behavior, perceptions, and technology unique to VWs. Hence, the question is, "How does collaboration in virtual worlds and the role of the facilitator change in comparison to real-world collaboration?"

Bringing an answer to this question allows organizations to train employees working in distributed teams to make better decisions, and form amiable virtual world relationships with partners and clients [19]. The current study aimed to shed light on this research question, and formulate a starting-point for researching the role of the facilitator in virtual world collaboration. Without a good understanding of the latter, organizational efforts to do business in such environments may fail and be perceived as unprofessional and/or a display of incompetence [29]. Such an understanding must be established before training facilitators to be effective in VWs. Further, the scope and capacity of virtual world collaboration is so vast that it would be difficult to have a trained facilitator readily accessible to most virtual groups. Consequently, it is likely that most virtual collaborations will be conducted by self-managed groups. With a firm understanding of what effective facilitation looks like in VWs, researchers can develop technology with embedded facilitation guidance for self-managed groups.

To examine the role of facilitation in VW collaboration, we developed interview questions concerning perceived collaboration opportunities afforded by VWs and how facilitators can get the most out of such opportunities. The questions were distributed to subject-matter experts who provided insights on best and worst VW facilitation practices. A cluster analysis of the responses from the participants revealed many considerations that must be made when facilitating in a VW. Based on the results, implications are discussed and future research directions identified.

\section{Background}

\subsection{Virtual world collaboration}

We focus on the use of Second Life in the current study as this is the most popular VW across the globe. Specifically, Second Life is a 3D virtual environment-referred to as a Multi-User Virtual Environment (MUVE) - in which one appears as an avatar and interacts with other people who are represented by their avatars [5, 25]. Like most MUVEs Second Life allows, users to customize their avatar, and exhibit just about any real-world behavior, such as making friends, interacting socially, shopping, vacationing, and doing business. Also, users can create and manipulate objects/artifacts in their environment ranging from images and tools, to their own island paradise.

MUVEs are expected to greatly enhance user engagement over traditional methods utilized by distributed groups. The primary driving force behind this assumption is that VWs are more engaging than traditional online communication [15, 35, 36]. This is particularly salient for collaborative team performance because engagement refers to a cognitive state in which a person is completely focused on a task [36]. When tasks are inherently engaging one's performance tends to increase [2], as does their propensity to experience another beneficial psychological state called flow [12]. Flow occurs when one reaches a state of enjoyment, satisfaction, and control resulting from being fully engaged in an activity [35]. In VWs, flow typically occurs due to a person's capability to co-exist in a realistic space where one can seemingly interact with others and the environment $[4,35]$. Specifically, being able to communicate (verbally and via text chat), exhibit emotions and body language, and express oneself via object creation, manipulation, and sharing makes for rich interactions.

Although little research has examined the effects of collaborating in different VWs, what has become a topic of exploration is the difference between collaborating in virtual environments in comparison to the physical world. Most of the major challenges inherent to VW collaboration have been identified as pertaining to limitations of the software, such as the absence of face-to-face communication and problems commonly associated with learning new and complex software. For instance, lack of face-to-face communication prevents group member from observing body language, having conversation with rich dialogue, and monitoring of participation [33, 44]. Such challenges in communication lead to social problems such as confusion, misunderstandings, 
interpersonal conflict, violation of group norms, and difficulty building trust between users [5, 37, 44].

Moreover, the technical challenges of VW collaboration further exasperate the complexities of virtual collaboration. VW software can put a heavy burden on a computer, which in-turn can cause unanticipated problems, such as operating system delays and shutdowns [6]. Such problems are frustrating for any user; however, technical difficulties cause the most problems for new users. The advantage of MUVEs is that they are powerful and malleable; however, for novice users, this is also a disadvantage as it can be difficult and time consuming to learn how to use the technology $[6,44]$. When users struggle to understand how to utilize VW software, both their performance and satisfaction with the technology dwindles [6]. Typically, discouraged users suffer from diminished self-efficacy-one's confidence in their ability to successfully perform a specific task [3]. Such users are likely to believe that they will not become a competent virtual person, and then justify that they do not need to improve their technical skills because VWs are just a game and not real work [5].

\subsection{Facilitation of collaboration}

By definition, 'to facilitate' literally means to 'make easy' [41, p.2]. In collaboration research, facilitation is typically considered to be the process by which "a person-whose selection is acceptable to all the members of the group, who is substantively neutral, and who has no substantive decision-making authority-diagnoses and intervenes to help a group improve how it identifies and solves problems and makes decisions, to increase the group's effectiveness." [38, p.3]. A strong body of research indicates that facilitation, compared to other collaboration techniques, helps teams yield higher quality outcomes, greater decision consensus, more efficient processes, greater cohesion, and greater participant satisfaction [1, 17, 20, 28, 43]. Furthermore, the tasks, styles, and characteristics by which facilitators deliver guidance influence team decision making outcomes. For instance, facilitators suggest which tools to use and when to use them [10, 42]. Such tools can range from simple pen and paper methods to advanced collaboration technologies with several settings and features.

Facilitation typically occurs as process or content facilitation. With process facilitation a facilitator acts impartial and only provides indirect contributions to a group's final solution or outcome by managing the process of communication and information processing by the group $[1,7,18]$. Process facilitation aims to ensure fair participation by all members. Conversely content facilitation occurs when facilitators actively help a group accomplish its goal by contributing knowledge, resolving conflicts, and providing a structure to the collaboration process [14]. Importantly, facilitation style matters as facilitators who are more dynamic, flexible, and adaptable tend to outperform those who are bound by structure to the point where they only stick to their scripts and do not customize their roles to the needs of the group [10]. Additionally, the costs and benefits of process versus content facilitation depend on the nature of the collaboration. For example, content facilitation tends to lead to faster solutions that carry greater group satisfaction, but this can also lead to overconfidence in a group [28]. Overconfident groups overestimate the contributions of the facilitator, fall victim to groupthink, and/or tend to generate fewer unique ideas and ultimately lower quality solutions because their members tend to trust that the facilitator helped find the right answer.

[30] conducted interviews to determine what characteristics a facilitator should have to effectively execute their tasks and styles. Respondents indicated that the two key qualities are good communication skills and ego-less facilitation, followed by understanding the group and its objectives, flexibility, task focus, and leadership. Interestingly, these characteristics are not always equally effective across situations. For instance, facilitators displaying transformational leadership are typically more effective than those displaying transactional leadership, but in VWs, the opposite is true [22, 39]. [22] suggested that charisma and individualized consideration-two major aspects of transformational leadership-are difficult to convey in VWs due to a lack of face-to-face communication.

Traditionally, the marriage between facilitators and collaboration technology has been positive and somewhat straightforward as effective facilitation techniques can be optimized by such tools [10, 17, 26]. Most commonly, facilitation techniques are paired with GSS to maximize the likelihood of high quality and satisfying collaborative outcomes. Therefore, research suggesting that interpersonal and leadership dynamics behind team collaboration might be different in VWs than in the real world is noteworthy.

\subsection{Overview of current study}

Although facilitation has been studied extensively in the real world, no studies have examined the role of the facilitators in VWs. Further, regardless of the medium, collaborative problem solving is challenging in any world - real or electronic. Most challenges of real world collaboration also occur in VWs because human beings will always face social and cognitive problems when they interact. Thus, the need to 
overcome the challenges inherent to group work is here to stay, and the complexity of these challenges is increasing with the sophistication of technology. As facilitators have successfully guided groups past such challenges in real world collaboration, organizations will rely on facilitators to do the same in VWs. Consequently, we must determine how facilitators can systematically replicate positive collaboration results from the real world in VWs. In doing so, we must understand both the challenges and best practices that VW facilitators are currently experiencing in the field.

\section{Method}

Fourteen subject-matter experts (10 males, 4 females) from around the world participated in the study. Participants' countries of residence included China, France, India, the Netherlands, and the US. Their expertise included many facets of facilitating technology supported collaboration, such as leadership, HCI, team decision making, GSS, and web-based systems. Participants had experience with VW collaboration using Second Life, Open Cobalt, Open Sim, Assemblive, World of Warcraft, and/or Ultima Online. Second Life was the only VW that all participants had experienced prior to the study; hence it is the focal VW of the current study. The sample included college professors and practitioners. Six professors who participated have taught a course using a VW.

An exploratory questionnaire addressing the use of facilitation in VWs was developed based on critical real world collaboration issues. A literature search of facilitation and collaboration studies revealed five general issues that need to be investigated in order to understand the role of the facilitator in VWs. First, we purport to identify what opportunities for collaboration exist in VWs. Second, we aim to better understand the differences and similarities of facilitating in a VW in comparison to the real world. Third, we examine what practices facilitators should implement and avoid to be effective in VWs. Fourth, we consider how effectively facilitation techniques can be taught in VWs. Fifth, facilitators' knowledge how to guide collaboration in VWs can have greater utility when paired with technology that supports collaborative efforts. Therefore, we investigated the strengths and weaknesses of using GSS to support VW collaboration.

Questions addressing these five themes were reviewed and revised by three subject-matter experts. The final questionnaire consisted of five open-ended questions that included sub-questions addressing specific aspects of the themes (e.g., strengths and weaknesses). An Internet and literature search was conducted to identify potential participants with practical and research experience in VW collaboration. The questionnaire was e-mailed to participants who were identified as having knowledge of both effective facilitation techniques and the use of VWs to conduct collaboration. Initially, a sample of convenience was targeted, as the researchers contacted participants with the aforementioned expertise. Participants were asked to provide referrals of other colleagues with similar expertise.

After receiving written responses and permission for further communication from participants, follow-up interviews were conducted via telephone or Skype when clarifications or further probing of responses was necessary. A sample size of 14 participants was deemed sufficient for this exploratory study as the no substantially new information was gained after collecting data from the $10^{\text {th }}$ participant.

Responses were de-identified and compiled into a database. A cluster analysis was conducted to categorize responses under similar general classifications. Category labels were created by paraphrasing the overarching theme of responses within each category. Responses that did not fit into a category were retained as independent ideas, but only uncategorized ideas that provided value beyond the extant categories are reported in the current study. The results from our qualitative analysis are provided in the next section.

\section{Results}

Q1: “What specific opportunities do VW environments provide for team collaboration?"

Respondents indicated that like most methods of online collaboration, VW collaboration can be utilized by anyone with internet access. Respondents also reported that like traditional methods utilized by distributed teams, such as teleconferencing and e-mail exchange, virtual teams enjoy the cost saving advantages of communication that does not require expensive travel arrangements. However, VW teams are unique from traditional distributed teams in that they allow for richer interaction. Respondents indicated that when VW teams interact, opportunities for increased social awareness arise, because being together in the same virtual space leads to enhanced perceptions of co-presence with others. For instance, respondents reported that associating a face with an interpersonal exchange seems to be more engaging than communicating solely via text or submitting work to a document sharing file. One respondent reported that during VW collaboration, "a participant can feel like (s)he is really working side-by-side with team 
members, as opposed to only contributing independent work to a nominal group."

Also, it was suggested that the ability to create and share objects adds a clarification dynamic to team interactions. Participants can better explain and understand concepts using visual modeling. Through object creation and manipulation, participants can simulate work processes and co-produce concepts. Such VW capabilities reduce misunderstandings relative to the use of e-mail or conference calls, because visual, spoken, and written communications can be used simultaneously to share an idea.

Respondents also mentioned that the opportunity for participating anonymously in VW collaboration can have its advantages. For example, participants may be more truthful and/or willing to contribute to group discussion when they do not have to face the evaluation apprehension associated with being judged by fellow group members. Also, anonymous idea contributions can improve group decision making by forcing team members to focus on the merits of ideas rather than who contributed them. Similarly, respondents suggested that being able to control the appearance of avatars may make participants more comfortable when interacting with a group because they can manipulate how they are seen. If group members believe that their personal appearances are unattractive, they may create an avatar in the image which they wish to be perceived. Thus, group members may become confident enough to participate in decision making when they do not fear others judging their demeaning or their physical appearance.

\section{Q2: How does the role of the facilitator change when interacting in a VW, and how does it remain the same?}

The role of the facilitator takes on new challenges in VW collaboration. First, VW collaboration is not truly face-to-face; rather, collaborators appear as avatars. Therefore, facilitators cannot read the body language of participants. This makes it more difficult to determine when a participant lacks understanding, is upset, or is not participating. Moreover, charisma is enacted differently in VWs than the real world. While real world facilitators can easily make gestures, expressions, and align voice inflections with body movements, a VW facilitator needs exceptional avatar management skills to be charismatic because they can only display non-verbal communication through manipulation of one's avatar. Lacking such skills may actually compromise a facilitator's credibility when working with experienced VW users.

A second challenge that facilitators face in VWs is that interpersonal interactions between participants can be more hostile. Respondents reported that miscom- munications are common in VWs, and that people can be mean when they know that they will never have to confront a team member in person. Interestingly, participants may face discrimination based on the physical characteristics of their avatars. Thus, real world social problems also appear in VWs, be it manifested differently. Respondents indicated that VW facilitators must be aware of these manifestations and take steps to prevent and alleviate such problems.

Specifically, respondents suggested that facilitators have to manage these challenges by providing clear instructions to the group and ensuring that the group understands the collaboration process. That is, with all of the distractions that can perspire in VWs, it is critical that facilitators clearly define the steps and outcomes of the collaboration process as well as the explicit roles of group members. Additionally, facilitators are responsible for asking participants if they need clarifications on the instructors or their roles, especially because facilitators cannot observe facial expressions or contextual reactions of collaborators.

Respondents were reluctant to make conjectures regarding what remains the same when facilitating collaboration in the real versus a virtual world. In fact, the majority of respondents did not answer the question or simply indicated that they did not know. Some hypothesized that the problem solving process will remain the same, i.e. they expect that sequences of collaborative activities that are effective in the real world to be just as effective in VWs. Additionally, respondents stated that VW facilitators will still have to deal with the same real world interpersonal conflicts and other problems associated with managing human interactions. They cited several of the aforementioned interpersonal conflicts such as more hostile confrontations and discrimination based on avatar appearance. A few new interpersonal problems that occur in both worlds were identified including social loafing, not considering others' points of view, and poor communication skills.

\section{Q3: What would you consider the best and worst practices that a facilitator could use in VW collaboration?}

In regards to best VW facilitation practices, respondents indicated that facilitators should first ensure that participants are proficient with the software being used and provide training resources for enhancing technical skills. Once a collaboration session begins, facilitators should evaluate whether each group member is in attendance and prepared to interact. Requiring members to introduce themselves both gets members acquainted and ensures that they are ready to participate. Additionally, many respondents indicated that it is imperative to set ground rules 
for communication before starting a collaborative session. Members should focus on being respectful when interacting with their team, keeping ideas focused on achieving the collective goal of the group, and following guidelines set forth by the facilitator.

Once participants are prepared to collaborate, a facilitator must ensure that communication is clear and efforts are focused toward the team's goal. One important method for keeping collaboration on track is to ask probing questions when necessary. Such questions might entail asking someone to re-phrase his idea. Facilitators can also ensure that members are paying attention by asking individuals specific questions, e.g. "Sam, what do you think about this?”.

Additionally, a VW collaboration process is likely to be new and uncomfortable for participants new to VWs. Consequently, it is important for facilitators to put collaborators at ease. On a basic level, this may involve providing encouragement to them and being approachable for questions. Creating a professional environment and providing participants with examples of successful VW collaboration projects may also make them more comfortable. Further, one respondent mentioned evidence from studies on the importance of having collaborators interact and get to know each other before beginning a session. Activities such as flying together or learning how to do a fun activity together builds group cohesion and improves outcomes more so than traditional "icebreakers" focused on learning the collaboration technology. Finally, at times participants may be insecure about interacting with other members, in which case it is important to emphasize that ideas can be shared anonymously.

Respondents also identified what they considered to be the most detrimental or worst practices when guiding VW collaboration. Respondents suggested that a facilitator could inhibit collaboration by creating an avatar with distracting physical characteristics. Creating a distracting avatar could provide some entertainment and momentarily increase team members' engagement, but in the long run it detracts attention from the problem at hand. A distracting avatar can make a facilitator appear less credible or serious and may even be offensive to some team members.

Another mistake is failing to provide software competence training, or more commonly, providing it during the session, rather than before the session. Training during the session was reported to be less effective because collaborators cannot learn at their own pace, and can be become overwhelmed by the thought that the session will be a waste if they do not learn the software quickly. Further, already proficient participants may become frustrated and distracted while waiting for novices to learn how to operate the tools. This frustration may lead to a bias against VW novices. Similarly, failing to arrange technical support may result in agitating users if a technical problem arises. More importantly, if a technical problem ends the session, time is lost, and team members may lose faith in the effectiveness of future sessions.

Once the session starts, a facilitator should not assume that everyone has the same proficiency in using the tools or understands what they are told to do. Instead, facilitators must be sensitive to individual differences, such as generational differences, eagerness to participate, dominance, and tendencies to not follow instructions or get side tracked. Some people may not be as comfortable using a computer or VWs as others, and people may have varying understandings of $\mathrm{VW}$ terminology. Respondents emphasized that VW facilitators must be perceptive of cues indicating that participants are uncomfortable or upset. For instance, novice users are less likely to have good avatar management skills, which can be reflected by their avatar facing the wrong way, a lack of non-verbal body movement or expressions from avatars, and a long response delay when asked a question. In all, the respondents suggested that in VWs, the facilitator must not take a passive role. Instead, the facilitator should focus more on encouraging novices to contribute to discussions, and instruct them how to do so.

\section{Q4: Could VWs be useful for training future facilitat- ors? What might be different from traditional training? \\ Respondents agreed that VWs are useful for train-} ing future facilitators. They described many ways in which VW training would be different from real world training. For instance, one respondent commented that "It is easier to get more practice in VWs because participants are more easily accessible than in the physical world." They also reflected on considerations to be made and challenges to be addressed in $\mathrm{VW}$ training. Most agreed that distance learning may be more difficult because a lack of face-to-face communication leads to the aforementioned social challenges of working in a VW. Specifically, respondents were concerned that "it is harder to check understanding and ensure that the right information is communicated and learned." Also, there is less accountability in VWs because instructors cannot be certain who is manipulating an avatar. Regardless of these benefits and challenges VW learners will need more technical skills than real world learners, such as preparation, installation, and execution of VW software.

\section{Q5: What are the greatest strengths and weaknesses of GSS based facilitation in virtual environments?}

Respondents indicated that utilizing GSS in VWs is advantageous because it is an easy to use, useful, and 
well established tool. When using GSS in VWs, team members do not have to install it on their computer; rather, the GSS appears on a virtual computer accessed by an avatar. Respondents also indicated that GSS and VWs are a good match because the advantages of both can be combined. For instance, using GSS provides a more comprehensive and accurate transfer of information in VWs than in the real world because all VW text conversations can be recorded. Also, screen shots and video recording can easily be taken of the collaboration process. Another important advantage of using GSS in VWs is that participants, not just their responses, can be truly anonymous. In the real world, idea contributions can be anonymous, but team member are still in proximity of one another. In VWs, team members may not know who is participating in a collaboration session, and open-discussions can be kept anonymous to the extent that team members only know what an avatar is saying. This anonymity may be particularly comforting to people who have a disability, fear discrimination based on physical appearance, or are participating in a session with someone they do not want to openly disagree with.

There are also reasons why using GSS in VWs may not be the best method for team decision making. Notably, some respondents suggested that VW use of GSS is "overkill" as they believe that it would be better to remotely plug into a traditional GSS. Others indicated that use of GSS via video conferencing would be better than having avatars perform GSS activities. Respondents were also concerned about data security. Due to the infancy of this technology, it is unclear how secure data is during sessions. Also, teams working with sensitive information are likely to fear putting confidential information online. Combining two technologies, GSS and VWs, also adds to the complexity of diagnosing technical and user problems encountered during a session. Finally, the complexity of the combined technologies may perpetuate the stigma that VWs are a place for geeks and social outcasts. Thus, business professionals may find it difficult to take the setting seriously.

\section{Discussion}

Past research has examined the role of the facilitator in guiding collaborative work practices. However, research on the role of the facilitator in VWs is still in its formative stages. This exploratory study investigates the role of the facilitator in $\mathrm{VW}$ collaboration as these environments yield new opportunities for facilitated collaboration. They are easier to access for remote group members located around the world. Also, VWs have important advantages over traditional collaboration in distributed teams as they support social awareness due to sharing the same virtual space, associating an avatar with a person, and actively working on a task along with other participants.

Although group interactions occur differently in VWs than in the real world, these interactions can still be guided by a facilitator. In considering how the role of the facilitator is different in VW environments, experts identified three primary challenges of facilitated collaborative problem solving in VWs.

First, interpersonal management is more difficult because non-verbal cues and attention focus cannot be monitored due to the absence of face-to-face communication. This limitation of VW collaboration makes it difficult for facilitators to identify participants in need of help. The same limitation prevents facilitators from determining when group members appear ready to move onto a new task-as evidenced by participants moving away from their keyboard or cracking jokes. Additionally, it is difficult to monitor participation and prevent social loafing, or recognize when people are displeased with the process or a group member. Notably, it is difficult for facilitators to show affection and build trust with participants who are not in the same physical environment. Facilitators may possibly overcome these challenges with exceptional avatar management and communication skills.

Second, with the introduction of more technology, more things can go wrong during a session. The complexity of combining technologies such as GSS with VWs can add to the list of complications associated with VW collaboration. Technical problems can paralyze a VW effort and discourage groups from future VW meetings. On a more basic level, when collaboration is conducted in a VW, not only do participants have to focus great cognitive effort on solving the problem, they also have to focus attention on avatar and GSS management. Thus, collaboration in VWs inherently causes high cognitive load, and it is the responsibility of the facilitator to put participants at ease with the system and help them focus their attention on the steps of the process.

Third, the appearance of a facilitator's avatar or avatar manipulation skills may be a source of discrimination, credibility loss, and/or loss of trust. Real world discrimination is not necessarily a product of proximity; rather it is based on a negative stereotype that automatically triggers negative reactions to a person's (or avatar's) physical characteristics [8]. Hence, in an effort to be entertaining, a facilitator may create an unusual or attention grabbing avatar and inadvertently inhibit the collaboration process. This process loss occurs because when a participant has an adverse perception of a facilitator's appearance, (s)he may not respect the facilitator enough to follow 
instructions, be too distracted to focus full attention on the problem at hand, or take the collaboration process less seriously. Similarly, poor avatar management may make a facilitator appear incompetent, and lose credibility. Respondents warned that if participants perceive facilitators to be incompetent or unprofessional, they will have difficulty building trust with participants due to limited opportunities for interactions. In the real world, if a facilitator gets off on the wrong foot with a participant, (s)he has more chances to rebuild that relationship via more frequent personal conversations and the opportunity to share emotions such as empathy or gratitude [13].

Respondents also identified four important steps facilitators can take to optimize VW collaboration. First, it is important to provide clear rules of communication and behavior. Being straightforward about how participants are expected to behave should reduce conflict and encourage positive problem solving behaviors. Introducing rules upfront puts participants at ease, and allows the facilitator to more easily manage conflicts by simply referring back to the rules. Second, facilitators should also ensure awareness of needed computer skills and proficiencies, as well as provide training resources prior to a session. Participants will not be able to adequately or at least confidently contribute if they are uncomfortable with the technology. Even experienced users can further hone their abilities and take full advantage of collaboration tools when facilitators provide technical training resources. Third, once a session is in progress, facilitators should provide encouragement throughout the session to put participants at ease with the software and process. Although facilitator involvement in the collaboration process is generally discouraged, VW facilitators must take a more active role in encouraging participants to overcome participation apprehension. It is critical that facilitators actively monitor participation so that they can determine when to assist those in need before they become too lost or frustrated. Fourth, facilitators must ascertain the technical skills necessary to effectively manipulate their avatars, utilize VW technology with grace, and trouble shoot technical problems. Such facilitators will avoid the embarrassment of looking like a rookie during a session or falling victim to technological malfunctions. They will also put participants at ease because participants will be comforted by the facilitator's confidence, ability to answer questions, and smooth operation of collaboration technology.

It is important to consider the limitations of the current study when evaluating or utilizing the aforementioned results and conclusions. First and foremost, the current study was an exploratory investigation; the results were based solely on personal experiences. The results were not based on empirical data or investigations of causal relationships. Additionally, the majority of respondents were only experienced in one specific VW, Second Life. Some experts we surveyed indicated that they also had experience facilitating meetings in Open Cobalt, Open Sim, and Assemblive. To date, no research has examined the differences between interacting in various types of VWs. It is important to acknowledge the context of respective virtual environments, and be mindful that behavior in one environment may not generalize to other environments.

Based on the results of the current study, we suggest four main research directions. First, it would be useful to explore the advantages and disadvantages of upcoming virtual environments other than Second Life. Do the functionality, usability, and popularity of other VWs have differential effects on collaboration processes and outcomes? For instance, would collaboration in a VW created by an organization for purposes of internal information sharing be taken more seriously and trusted more than collaborating on the same project in Second Life?

Also, we must examine what factors moderate the relationship between facilitator behaviors and effectiveness in virtual environment collaboration. For example, does empirical evidence support the qualitative finding from the current study suggesting that facilitators with good technical skills are more charismatic, trustworthy, engaging, and effective?

Similarly, the current exploratory study indicated that the role of the facilitator changes in VWs, and that facilitators must capitalize on new best practices while avoiding pitfalls unique to a virtual environment. These assertions must be tested in an empirical study geared toward contrasting the role and effectiveness of the facilitator in virtual versus physical environments. It is possible that participant reactions to and outcomes from facilitated collaboration in VWs were only perceived or expected to change, and objective measures may indicate that this was not the case. Instead, VW facilitation roles may be very similar to real world facilitation roles, and new practices may not improve VW collaboration.

Finally, the current study examined the opinions and perceptions of facilitators and experienced researchers. We must also assess participants' perceptions of virtual environment facilitators, as well as how these facilitators are perceived in relation to physical world facilitators. Tapping into the perceptions of participants could help identify what individual differences or personal attributes make facilitators more or less effective. Participants can also indicate if they find that specific facilitator behaviors increase their engagement in collaborative problem 
solving. For instance, a facilitator might think that teaching the virtual mamba to participants as an icebreaker may make him appear more charismatic and enhance the effectiveness of the group. However, a participant may disagree and suggest that the facilitator's mamba routine was a childish distraction.

\section{Conclusion}

Our exploratory study found that a sample of collaboration experts have both enjoyed success and endured unexpected challenges in facilitating VW collaboration. Initial evidence indicates that the role of the facilitator does in fact differ between virtual worlds and the real world. It seems that the absence of face-toface communication and accountability, in particular, creates new social problems that facilitators will have to overcome during VW collaboration efforts. As such, future interventions aimed at managing $\mathrm{VW}$ challenges will be paramount to the success of VW collaboration.

Participants indicated that some established realworld collaboration practices do seem to effectively translate to VWs. The design of decision making strategies, such as brainstorming, convergence, and idea evaluation techniques seem to be just as useful in VWs as in the real-world. However, participants were reluctant to declare similarities in facilitation across worlds as interpersonal exchanges in VWs reportedly occur differently enough that participants found human behavior more difficult to predict. Future studies are needed to quantitatively assess the effectiveness of real-world facilitation strategies in virtual worlds.

The current study helps to shape a research agenda for determining how facilitation and collaborative decision making can be optimized in VWs. With increasing globalization and the speed of technological development-especially in regards to online activities-VWs are likely to become more user friendly, powerful, and popular in the relatively near future. Therefore, more in-depth and quantitatively driven studies examining the assertions made by our participants can shape the future on VW collaboration.

\section{References}

[1] R. Anson, R. Bostrom, and B. Wynne, “An Experiment Assessing Group Support System and Facilitator Effects on Meeting Outcomes”, Management Science, 1995, pp. 189-208.

[2] A. Bakker, S. Albrecht, and M. Leiter, "Key questions regarding work engagement”, European Journal of Work and Organizational Psychology, 2011, pp. 4-28.

[3] A. Bandura, "Fearful expectations and avoidant actions as coeffects of perceived self-inefficacy", American Psychologist, pp. 1389-1391.
[4] R. Bartle, Designing Virtual Worlds. New Riders Games, 2003.

[5] K. Bessier, J. Newhagen, Robinson, and B. Shneiderman, "A model for computer frustration: The role of instrumental and dispositional factors on incident, session, and post-session frustration and mood”, Computers in Human Behavior, pp. 941-951

[6] W. M. Bulkeley, "Playing well with others", Wall Street Journal, June $18^{\text {th }} 2007$.

[7] K. Bessiere, J. Ellis, and W. Kellogg, "Acquiring a professional Second Life: Problems and prospects for the use of virtual worlds in business", In Proceeding of the twenty-seventh annual SIGCHI conference on Human factors in computing systems. 2010.

[8] R. Bostrom, R. Anson, V. and Clawson, V.K. 1993. "Group facilitation and group support systems", Group Support Systems: New Perspectives, Jessup, L.M. and Valacich, J.S., eds. New York: Macmillan, p.146-148

[9] Current user metrics for Second Life, Retrieved 201002-19, 2010.

[10] G. Dickson, M. Limayem, J. Lee Partridge, G. DeSanctis. "Facilitating computer supported meetings: A cumulative analysis in a multiple criteria task environment”, Group Decision and Negotiation, 1996, pp. 51-72.

[11]M. Diehl, \& W. Stroebe, "Productivity loss in ideagenerating groups: Tracking down the blocking effect", Journal of Personality and Social Psychology, 1987, pp. 392-403.

[12] E. Driver, C. Moore, C. Schooley, \& J. Barnett, "Getting real work done in virtual worlds", Forrester Research Reports, Forrester Research, Inc., 2008 January 7th.

[13] E. Driver, P. Jackson, T. Keitt, C. Moore, C. Schooley \& J. Barnett, "Web 3D: The next major internet wave", Forrester Research Reports, Forrester Research, Inc., 2008, April $18^{\text {th }}$.

[14] C. Eden. "The unfolding nature of group decision support: Two dimensions of skill,” in Tackling Strategic Problems-The Role of Group Decision Support. C. Eden and J. Radford (Eds.), Sage Publications, London, 1990.

[15] K. Franceschi, R. Lee, and D. Hinds, "Engaging elearning in virtual worlds: Supporting group collaboration", Proceedings of the $41^{\mathrm{st}}$ Annual Hawaii International Conference on System Sciences (2008), 7

[16] S. Gaudin, "3D internet forecast within 5 years", Computerworld, 2010, April 11th., pp.

[17] J. George, A. Dennis, and J. Nunamaker. “An experimental investigation of facilitation in an EMS decision room”, Group Decision and Negotiation 1992, pp. 57-70.

[18] T. Griffith, M, Fuller, G. Northcraft,. "Facilitator influence in group support systems: Intended and unintended effects", Information Systems Research, 1998, p. 20-36.

[19] A. Hendaoui, M. Limayem and C. W. Thompson, "3D xocial virtual worlds: Research issues and challenges", IEEE internet computing, (2008, Jan/Feb), pp. 88-92.

[20] T. Hostager, S. Lester, K. Ready, and M. Bergmann. "Matching facilitator style and agenda structure in group support systems: Effects participant satisfaction and 
group output quality", Information Resources Management Journal, 2003, pp. 56-62.

[21] C. R. Jones, \& R.H. Fazio, "Person categorization and automatic racial stereotyping effects on weapon identification", Personality and Social Psychology Bulletin, 2010, pp. 1073-1085.

[22] S. Kahai and B. Avolio, "Effects of leadership style and anonymity on the discussion of an ethical issue in an electronic meeting system context”, in Leadership at a distance: Research in technologically-supported work S. P. Weisband (Ed.) , Lawrence Erlbaum Associates Publishers,.Mahwah, NJ, 2008, pp. 97-125.

[23] E. C. S. Kahai,and R. Jestice, "Team collaboration in virtual worlds", DataBase, 2007, pp. 61-68.

[24] O. Kharif, "The virtual meeting room", BusinessWeek, Retrieved from http://www.businessweek.com/technology/content/apr2007/tc20070416_445840.htm, 2007.

[25] F. Li, S. Papagiannidis, and M. Bourlakis, "Living in 'multiple spaces': extending our socioeconomic environment through virtual worlds", Environment and Planning D: Society and Space, 2010, pp. 425 - 446.

[26] M. Limayem, J. Lee-Partridge, G. Dickson, and G. DeSanctis, "Enhancing GDSS effectiveness: Automated versus human facilitation”, Proceedings of the $26^{\text {th }}$ Hawaiian International Conference on System Sciences.

[27] Linden Labs, http://work.secondlife.com/enUS/successstories/, 2009.

[28] S. Miranda, R. Bostrom, "Meeting Facilitation: Process Versus Content Interventions", Journal of Management Information Systems, 1999, pp. 89-114.

[29] G. Moscarini, "Competence implies credibility", American Economic Review, 2007, pp. 37-63.

[30] F. Niederman, C. Beise, and P. Beranek, "Issues and concerns about computer-supported meetings: The facilitator's perspective”,Management Information Systems Quarterly, 1996, pp. 1-22.

[31] K. Nielsen, \& B. Cleal, "Predicting flow at work: Investigating the activities and job characteristics that predict flow states at work", Journal of Occupational Health Psychology, 2010, pp. 180-190.

[32] T. S. Larey, and P.B. Paulus, "Group preference and convergent tendencies in small groups: A content analysis of group brainstorming performance", Creativity Research Journal, 1999, pp. 175-184.
[33] G. Olson and J. Olson, "Distance matters", Human Computer Interaction, 2000, pp. 139-179.

[34] L. Peters, and R.J. Karren, "An examination of the roles of trust and functional diversity on virtual team performance ratings", Group \& Organization Management, 2009, pp. 479-504.

[35] A. Quinn, "An exploratory study of opinions on clickers and class participation from students of human behavior in the social environment", Journal of Human Behavior in the Social Environment, 2010, pp. 721-731.

[36] K. Salen and E. Zimmerman, "The rules of play.", MIT Press, Cambridge, MA, 2004.

[37] R. Schroeder, "The social life of avatars: Presence and interactions in shared virtual environments", Proceedings of the 11th Annual International Workshop on Presence, Padova, 16-18 October 2008.

[38] R. Schwarz, "The Skilled Facilitator: A Comprehensive Resource for Consultants, Facilitators, Managers, Trainers, and Coaches", Jossey Bass, New York, 2002, Springer Verlag, Berlin, 2002.

[39] J. Sosik, B. Avolio, B, and S. Kahai, "Effects of leadership style and anonymity on group potency and effectiveness in a group decision", Journal of Applied Psychology,, Feb 1997, pp. 89-103.

[40] D. Takashashi, "Q\&A: Linden Lab CEO Mark Kingdon on Second Life's latest evolution", Social Beat: Interpreting Innovation, 2008, September $18^{\text {th. }}$

[41] J. Townsend and P. Donovan, "The facilitator's pocketbook", Management Pocketbooks, Hants, UK, 1999.

[42] G. J. de Vreede, ,J. A. Boonstra,, and F. Niederman, "What Is Effective GSS Facilitation? A Qualitative Inquiry Into Participants’ Perceptions”, in Proceedings of the 35th Hawaiian International Conference on System Sciences, IEEE Computer Society Press.,2002.

[43] B. Wheeler, J. Valacich, "Facilitation, GSS, and training as sources of process restrictiveness and guidance for structured group decision making”, Information Systems Research, 1996, pp.429-450.

[44] N. Yee, J. Bailenson, M. Urbanek, F. Chang, and D. Merget, "The unbearable likeness of being digital: The persistence of nonverbal social norms in online virtual environments", The Journal of CyberPsychology and Behavior, 2007, pp. 115-121. 